\title{
Sobre a Aquisição Tardia de QU IN Situ em Português Brasileiro*
}

\author{
(On the Late Acquisition of Wh In Situ \\ in Brazilian Portuguese)
}

Elaine Grolla

(University of Connecticut)

\begin{abstract}
In this paper I am concerned with the acquisition of WH in situ questions in Brazilian Portuguese. I try to account for the fact that the child being studied here starts producing this type of question relatively late, if compared to the production of other structures occurring at the left periphery of the sentence. This fact is supprising, given that in adult language such constructions are fairly frequent (cf. Lopes-Rossi (1996)). I present an analysis to the child's data claiming that the child changes her initial setting of the parameter regulating the operation of WH movement. Thisparameter resetting is triggered by the identification of a cue present in the input. Such parameter resetting is argued to be necessary to properly account for the acquisition data.
\end{abstract}

KEY-WORDs: language acquisition; wh-in situ questions; cue-based theory.

RESUMO: Este estudo analiza a aquisiz̧ão de interrogativas com o elemento $Q U$ in situ em Português Brasileiro. Tento dar conta do fato de a crianşa produzir tais interrogativas muito tardiamente, se comparado com a produção de outros fenômenos que ocorrem na periferia esquerda da sentença. Tal fato é considerado surpreendente, uma vez que foi constatado em outros trabalhos que, em PB adulto falado, tais construçôes são relativamente frequientes (cf. Lopes-Rossi (1996)). Apresento uma análise para os dados da criança, sugerindo que no curso da aquisição a criança altera a marcaşão inicial do parâmetro que regula esta operação. Tal remarcaşão é desencadeada pela identificaçãa de uma pista presente no input. Concluo que a postulaşão de tal remarcaşão é crucial se quisermos explicar os dados de aquisiçãa apropriadamente.

PALAVRAS-CHAVE: aquisiz̧ão de linguagem; perguntas-QU in situ; teoria baseada em pistas.

* Gostaria de agradecer a Maria Cecília Perroni, Jairo Morais Nunes, Ruth Lopes, Marcelo Barra Ferreira e a dois revisores da revista pelas sugestões, críticas e comentários. Agradeço também à FAPESP pelo apoio financeiro.

D.E.L.T.A., 21:1, $2005(57-73)$ 


\section{Introdução}

Este trabalho trata da aquisição de perguntas com o elemento QU in situ em Português Brasileiro (doravante $\mathrm{PB}$ ) na fala de uma criança (N.) adquirindo o PB como língua nativa. ${ }^{1}$ Veremos que os dados de aquisição podem ajudar a clarificar questões sobre o $\mathrm{PB}$ adulto que ainda são bastante debatidas por estudiosos desta língua. Como será mostrado adiante, os dados de N. são bastante diferentes quantitativamente dos dados do PB adulto: enquanto que no adulto existem estudos que indicam que há uma produção significativa de perguntas com elemento QU in situ (cf. LopesRossi (1996)), nos dados infantis notamos uma quase que completa ausência dessa construção.

Além de escassas, as primeiras perguntas que envolvem esta construção emergirão na fala de $\mathrm{N}$. somente depois dos 3 anos e 9 meses de idade, o que pode ser considerado uma aquisição tardia, já que outras estruturas contendo dependências-A' (tais como perguntas clivadas, pseudo-clivadas, orações relativas e contruções de tópico-comentário) emergiram na fala da criança bem antes desta idade. ${ }^{2}$ Dito isto, as perguntas que imediatamente nos vêm à mente podem ser formuladas da seguinte maneira: (a) por que a aquisição desta construção é tão tardia, relativamente às demais estruturas envolvendo dependências- $A$ "? (b) de que forma a criança "aprende" que a gramática de sua língua pode gerar QU in situ?

Para responder estas questões, assumirei a 'teoria baseada em pistas' (ingl. 'Cue-based theory') explorada em Dresher e Kaye (1990), Dresher (1999), Fodor (1998) e Lightfoot (1999) que propõem que a Gramática Universal (GU) especifica não somente um conjunto de parâmetros, mas para cada parâmetro uma pista. Esta pista seria um tipo de estrutura, um elemento da gramática, que seria derivada do input e que seria encontrada nas representações mentais que resultam da escuta, entendimento e decodificação dos enunciados. Mais adiante apresentarei detalhadamente esta teoria e analisarei os dados de $\mathrm{N}$. e os dados do PB adulto com o intuito de

\footnotetext{
1 Esta pesquisa foi realizada com base em dados do Projeto sobre Aquisisção de Linguagem do Departamento de Lingüística, IEL, Unicamp. Eles totalizam 53 sessões de gravação em áudio-tape de N., que foi gravada entre os $2 ; 0$ e os $4 ; 0$ de idade. Trata-se de um estudo longitudinal, observacional.

2 Dados apresentados em Grolla (2000).
} 
detectar uma pista (ou várias pistas) que possa(m) explicar como o comportamento lingüístico da criança se modificou no curso de aquisição de perguntas QU nesta língua. Tentarei dar conta do fato de que, num primeiro momento, a gramática da criança não gerava estruturas com QU in situ e, num momento posterior, a geração desta estrutura é licenciada.

O artigo está dividido da seguinte forma: na seção 1 apresentarei a tipologia proposta por Cheng (1991) para classificação das línguas em línguas de QU in situ ou em línguas de movimento QU. Kato e Raposo (1994) propõem uma análise para o $\mathrm{PB}$ parecida com a sugestão de Cheng. Por isso, apresento a proposta desses autores na mesma seção. Na seção 2, veremos que existe uma análise alternativa para darmos conta não só do PB adulto, como também dos dados infantis, o que pode nos auxiliar a responder as perguntas (a) e (b) feitas acima. $\mathrm{Na}$ seção 3, apresento os dados de aquisição detalhamente e os comparo aos dados do PB adulto apresentados em Lopes-Rossi (1996). Na seção 4, os dados de aquisição são discutidos com base na análise feita na seção 3. Por fim, na seção 5, é apresentada a conclusão deste estudo.

\section{Cheng (1991) e Kato e Raposo (1994)}

Cheng (1991) propõe uma tipologia das línguas naturais, separandoas em dois grupos: as línguas de QU in situ e as línguas de movimento QU. As primeiras nunca efetuam movimento do elemento QU e as línguas do segundo tipo nunca deixam o elemento interrogativo em sua posição argumental. Além disso, a autora também apresenta uma análise para algumas línguas que possuem o elemento QU in situ apenas opcionalmente, fato que ocorre também em PB. Kato e Raposo (1994) propõem uma análise para algumas interrogativas do $\mathrm{PB}$ que se aproxima bastante da proposta de Cheng para as línguas com QU in situ opcional. Por isso, analisarei a proposta de Cheng em mais detalhes e considerarei quais as vantagens e desvantagens que a adoção desta proposta traz para uma teoria que pretenda explicar a aquisição das perguntas com QU in situ em PB.

Cheng (1991) adota a 'hipótese da classificação de oração' (ingl. 'Clausal Typing Hypothesis') que requer que todas as orações sejam classificadas em estrutura-S (Chomsky e Lasnik (1977)). Ou seja, cada oração deve ser identificada com um dos 'tipos de sentença', como: interrogativa, declara- 
tiva e, em algumas línguas, quotativa, presumptiva, etc. Para explicar como isso se dá no que concerne às interrogativas, Cheng, baseada em dados de línguas como o chinês, o japonês e o inglês, propõe que as línguas que possuem uma partícula de pergunta (tanto em perguntas QU como em perguntas $\operatorname{sim}$ /não) classificam as sentenças interrogativas utilizando tal partícula. Já nas línguas sem esta partícula, as palavras QU têm de efetuar movimento para classificar as sentenças como interrogativas. Observe as sentenças abaixo, como exemplos de interrogativas em inglês e chinês (dado do chinês retirado de Cheng 1991:30):

(1) a. $\left[_{\mathrm{CP}}\right.$ What ${ }_{\mathrm{i}}\left[_{\mathrm{TP}}\right.$ did you buy $\left.\mathrm{t}_{\mathrm{i}}\right]$ ?

$\mathrm{O}$ que você comprou t

b. Qiaofong mai-le sheme ne

Qiaofong compra-ASP o que $\mathrm{Q}_{\mathrm{QU}}$

$\mathrm{O}$ que Qiaofong comprou?

Além disso, Cheng mostra que no japonês, por exemplo, as palavras QU podem ser interpretadas como interrogativas, existenciais e universais, pois elas não possuem força quantificacional inerente. Numa pergunta QU, a leitura interrogativa da palavra QU é determinada pela partícula interrogativa. Essa partícula é gerada em $\mathrm{C}^{\circ}$ e está presente não só nas perguntas QU como também nas perguntas sim/não. A forma destas partículas varia de língua para língua e pode variar até mesmo na mesma língua. Em mandarim, por exemplo, a partícula que aparece em perguntas sim/não tem a forma ' $m a$ ', já a partícula que está presente em interrogativas QU tem a forma ' $n e$ ', ou é nula. ${ }^{3}$ Cheng apresenta então a seguinte generalização (Cheng 1991: 24):

(2) "Línguas in situ têm partículas QU. Línguas com partículas QU são línguas in situ."

As línguas que não dispõem desta estratégia de inserção de partículas classificam suas interrogativas com o movimento QU. Portanto, tal movimento se mostra como uma operação do tipo 'último recurso', ocorrendo somente nos casos em que a partícula não pôde ser inserida durante a derivação. As línguas que possuem a partícula interrogativa não efetuam

3 Cheng observa que, nas línguas de QU in situ, a partícula interrogativa nunca é nula nas perguntas sim/não, mas pode ser nula nas perguntas QU, como é o caso do chinês. 
movimento da palavra QU, uma vez que a inserção da partícula sempre será menos custosa que a operação de movimento. A sua proposta pode então ser resumida da seguinte forma (Cheng 1991: 30):

(3) “Toda oração precisa ser classificada. No caso de se classificar uma pergunta $\mathrm{QU}$, tanto uma partícula QU em $\mathrm{C}^{\circ}$, quanto o alçamento de uma palavra QU para o Spec, CP podem ser usados."

Um eventual contra-exemplo para a teoria de Cheng seria uma língua que tem as duas opções: ora deixa a palavra QU in situ (usando uma partícula para classificar a sentença), ora move uma palavra QU em estrutura-S. Com base na generalização apresentada acima, como explicar o fato de que existem línguas que, mesmo possuindo a partícula interrogativa, dispõem das construções com QU deslocado? Cheng analisa três línguas que apresentam esta característica: o árabe egípcio, o bahasa indonesia e o palauan.

Nessas três línguas, a autora observa que as interrogativas com o elemento QU deslocado são construídas com um complementizador realizado abertamente em $\mathrm{C}^{\circ}$. O complementizador utilizado em interrogativas é o mesmo complementizador empregado em relativas e clivadas e difere do complementizador que aparece em subordinadas regulares. Além da similaridade na forma do complementizador, as condições sobre efeitos de ilhas são compatíveis tanto nas interrogativas como nas clivadas. Baseada neste tipo de evidência, a autora propõe que estas línguas possuem orações interrogativas de dois tipos: (a) interrogativas com QU in situ e (b) interrogativas com o elemento QU aparecendo na periferia esquerda. Neste último caso, não haveria movimento QU para a periferia esquerda. Ao invés disso, essas interrogativas seriam derivadas de orações clivadas reduzidas. Dessa forma, a opção de movimento QU nunca estaria disponível, sendo que as construções envolvendo o elemento interrogativo na periferia esquerda teriam este elemento gerado na base diretamente nesta posição. Em (4a) abaixo, temos a estrutura de uma sentença clivada em Inglês. Em (4b) temos a estrutura de uma clivada reduzida em árabe egípcio (dado retirado de Cheng 1991:64):

(4) a. Estrutura da clivada completa It is $\left[_{C P}\left[_{D P}\right.\right.$ Sharon $]\left[_{C P}\right.$ OP ${ }_{i}$ that $\left[_{I P}\right.$ Marcia likes $\left.\left.\left.t_{i}\right]\right]\right]$ É Sharon que Marcia gosta 
b. Estrutura da clivada reduzida

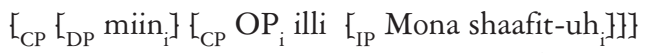

quem que Mona viu-ele

'Quem que a Mona viu?'

Esta análise é estendida para o bahasa indonesia e o palauan, uma vez que estas línguas também apresentam as similaridades entre clivadas e perguntas QU existentes em árabe egípcio. Dessa forma, os potenciais contra-exemplos à proposta de Cheng seriam, na verdade, línguas que não dispõem da operação de movimento QU; quando os elementos interrogativos aparecem na periferia esquerda, eles estão lá devido a algum outro tipo de operação que não movimento.

Kato e Raposo (1994), investigando algumas diferenças entre o PB e o Português Europeu, propõem uma análise para o PB parecida com a análise de Cheng para o árabe egípcio. Os autores assumem que as interrogativas do tipo: "Quem me deu o CD?" seriam derivadas das orações clivadas. Observe as etapas desta derivação abaixo (retirado de Kato e Raposo 1994: 273-274):

(5) a. Estrutura da oração clivada:

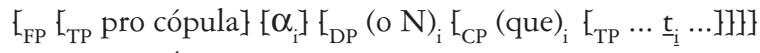
pro é quem que .....

b. Alçamento aberto do foco para spec de FP da oração matriz: Quem é t que me deu o CD?

c. Regra de apagamento de cópula em construções clivadas:

Quem que me deu o CD?

d. Apagamento do complementizador que:

Quem me deu o CD?

Se assumirmos que esta análise está no caminho certo e estendemo-la para todas as interrogativas QU em $\mathrm{PB}$, poderíamos classificar o $\mathrm{PB}$ como uma língua que nunca dispõe de movimento QU para a periferia esquerda, sendo que os casos de QU deslocado constituiriam na verdade interrogativas derivadas de clivadas, em que nenhum movimento de dentro de TP para a periferia esquerda tenha ocorrido. A vantagem desta análise seria que, do mesmo modo que nas línguas estudadas por Cheng, as interrogativas em PB possuiriam (opcionalmente) um complementizador realizado abertamente em $\mathrm{C}^{\circ}$, exatamente como nas clivadas e relativas desta língua. Além disso, teríamos uma análise mais uniforme, já que poderíamos 
analisar o PB como uma língua que possui o valor paramétrico [-movimento QU], explicando a presença de perguntas com QU in situ.

No entanto, esta análise apresenta alguns problemas. O primeiro deles é que, diferentemente das línguas estudadas por Cheng, o PB não dispõe de partícula interrogativa em suas perguntas. Segundo a generalização apresentada acima, a língua que não possui partícula interrogativa não pode ser classificada como uma língua de QU in situ. Se assumirmos que esta partícula está presente, mas é sempre nula, enfraquecemos a proposta de Cheng, uma vez que a autora não baseia sua hipótese em nenhuma língua com partícula interrogativa nula tanto em perguntas QU como em perguntas sim/não. Além disso, como explicaríamos a aquisição desta estrutura? A criança não teria nenhuma evidência independente para postular a existência de tal partícula.

Além disso, um outro problema para esta análise é o fato de que, durante o processo de aquisição do $\mathrm{PB}$, a criança começa a produzir orações interrogativas com QU deslocado muito tempo antes de produzir a primeira sentença clivada. Ou seja, como explicar que já aos 2;3 de idade a criança estudada aqui apresentou uso produtivo de perguntas do tipo "o que você quer?" mas ainda não tenha produzido nenhuma oração do tipo "o que é que você quer?" que, teoricamente, seria a origem da primeira pergunta e que surge somente aos 3;2 de idade nos dados de N.? Do ponto de vista da aquisição, seria um tanto quanto não natural postular este tipo de análise, uma vez que teríamos que assumir que a criança adquire uma estrutura derivada antes mesmo de adquirir a estrutura que a origina. Por esses fatos, acredito que devemos procurar uma outra forma de analisar tanto os fatos do PB adulto quanto os dados da criança.

\section{O PB como língua [+ movimento QU] opcional}

Vimos na seção anterior que tentar classificar o PB como uma língua de QU in situ traz problemas tanto descritivos como explicativos. Segundo a proposta de Cheng apresentada acima, não podemos admitir uma análise que classifique o PB como língua de QU in situ, uma vez que não há partícula interrogativa nesta língua. Por outro lado, se o PB fosse uma língua com o traço [+ movimento QU], não apresentaria perguntas com QU in situ. Ou seja, na teoria de Cheng, o PB não se qualifica nem como língua de QU in situ nem como língua de movimento QU. 
De acordo com a proposta minimalista (Chomsky (1995)), traços fortes devem ser eliminados da derivação antes de Spell-out e traços fracos devem ser checados depois de Spell-out, no componente coberto. Traduzindo a proposta de Cheng em termos de traços, as línguas com partículas interrogativas sempre possuem o traço [QU] fraco, já que o movimento QU é coberto. Por outro lado, as línguas que efetuam movimento QU possuem o traço QU sempre forte, uma vez que tal movimento tem de ser efetuado abertamente. O comportamento do PB neste contexto é determinante para assumirmos que esta divisão das línguas em 'língua com traço sempre forte' e 'língua com traço sempre fraco' não pode ser tão rígida. Temos de enfraquecer esta divisão para dar conta dos fatos do PB. Proporei então que o traço [QU] presente nos sintagmas interrogativos em $\mathrm{PB}$ pode ser tanto forte como fraco. Se for forte, obteremos perguntas como em (6) abaixo. Se, por outro lado, este traço for fraco, perguntas como em (7) emergirão:

(6) a. Quem você encontrou t?

b. O que o João queria t?

c. Como você chegou lá t?

(7) a. Você encontrou quem?

b. O João queria o que?

c. Você chegou lá como?

Em (6), as palavras QU se moveram para spec, $\mathrm{CP}$ para checar o traço forte em estrutura-S. Em (7), no entanto, como o traço QU é fraco, ele somente será checado em Forma Lógica(FL), de acordo com Chomsky (1995).

Embora esta análise possa dar conta do PB adulto, como explicar a aquisição dessas estruturas? Ou mais explicitamente: como a criança saberá que sua língua tem traço [QU] forte opcional? Na próxima seção descrevo os dados de $\mathrm{N}$. mais detalhamente.

\section{Os dados de aquisição}

Até os 3;9 de idade, N. produz apenas perguntas com o elemento QU deslocado, como abaixo: ${ }^{4}$

\footnotetext{
4 Nos enunciados de N. apresentados no texto, os números entre parênteses indicam a idade da criança (em (anos; meses)) quando tais sentenças foram gravadas.
} 
(8) a. Que é isso aqui?

b. Dõndi vai?

c. Porque você tá fazendu assim?

d. Qual que o pai fez?

Somente depois dos 3;9, as perguntas com QU in situ aparecem: ${ }^{5}$

(9) a. Criança: Que hora que é?

Aduto: $\quad 14$ minutos para as 17 horas.

Criança: Pái aonde?

Aduto: $\quad$ Não. Falta 14 minutos.

Criança: Páía...aondi?

Aduto: Embora.

Criança: Aon ...pá onde?

b. Criança: Quê isso?

Aduto: $\quad$ É fio.

Criança: Pá fazer o quê?

c. Eu quero brincar com quê??

d. Parece quem? Hãn? Parece quem da novela?

e. Eu tô brincando sabe com quem?

Pelo quadro abaixo podemos notar que há um baixíssimo uso de perguntas com QU in situ, constituindo apenas 1,7\% do total de perguntas.

(10) Tabela com o tipo de pergunta encontrado no corpus de N. ${ }^{6}$

\begin{tabular}{l|c|c}
\hline Tipo de pergunta & Ocorrências & Porcentagem \\
\hline Q deslocado & 344 & $66 \%$ \\
\hline Q - que & 153 & $29,4 \%$ \\
\hline Q - é que & 15 & $2,9 \%$ \\
\hline Q in situ & 08 & $1,7 \%$ \\
\hline Total & 520 & $100 \%$ \\
\hline
\end{tabular}

\footnotetext{
5 Embora algumas das construções de QU in situ apresentadas em (9) não se alternem com uma interrogativa com QU deslocado, para os nossos propósitos continuaremos considerando que todas elas sejam verdadeiras interrogativas com QU in situ.

6 Neste quadro, foram retiradas as perguntas QU envolvendo o elemento interrogativo 'quem' com a função sintática de sujeito, uma vez que nessas estruturas não podemos ter certeza sobre a ocorrência de movimento para a periferia esquerda ou não. A célula "Que - que" se refere às perguntas do tipo: 'o que que você quer?'. A célula "Que - é que” se refere às perguntas do tipo: 'o que é que você quer?'.
} 
Esses números são bastante diferentes dos dados do adulto, como mostrado em Lopes-Rossi (1996). No quadro abaixo forneço o número de perguntas QU no PB adulto falado. Para uma melhor visualização do contraste entre os números encontrados na fala adulta e na fala da criança, forneço na coluna sombreada as porcentagens do quadro (10) acima (dados da língua adulta retirados de Lopes-Rossi 1996: 117):

(11) Tabela com perguntas QU em PB adulto falado versus a produção de N.

\begin{tabular}{l|c|c|c}
\hline Tipo de pergunta & NURC & TV & Criança \\
\hline Q deslocado & $29 \%$ & $30 \%$ & $66 \%$ \\
\hline $\mathrm{Q}-$ que & $21 \%$ & $19 \%$ & $29,4 \%$ \\
\hline $\mathrm{Q}-$ é que & $37,5 \%$ & $18,6 \%$ & $2,9 \%$ \\
\hline $\mathrm{Q}$ in situ & $12,5 \%$ & $32,4 \%$ & $1,7 \%$ \\
\hline Total & $100 \%$ & $100 \%$ & $100 \%$ \\
\hline
\end{tabular}

É importante observar que os dados de N. são parecidos com os dados de três crianças estudadas em Sikansi (1999). Esta autora investiga os dados de 3 crianças adquirindo o PB como língua materna. Tais dados corroboram os números encontrados na presente pesquisa: nos dados das três crianças estudadas em Sikansi (1999) houve apenas duas manifestações de QU in situ num total de 209 perguntas.

Podemos dizer então que crianças adquirindo o PB usam muito marginalmente as construções com QU não deslocado. Os dados aqui apresentados, juntamente com os dados de Sikansi, mostram que a aquisição de QU in situ é lenta e difere quantitativamente do que é encontrado na gramática do PB adulto.

O nosso problema é então explicar como durante muitos meses (até mesmo durante anos), a criança produz apenas estruturas com movimento e, a partir de um dado momento, perguntas com o elemento interrogativo in situ começam a ser produzidas. Na próxima seção, tentaremos explicar este comportamento da criança. Veremos que a análise que toma o PB como língua com traço [QU] forte opcional é corroborada pelos dados de aquisição. 


\section{A aquisição de $\mathrm{QU}$ in situ}

Para dar conta das indagações feitas na seção anterior, assumirei que, no processo de aquisição de uma língua, a criança se utiliza de pistas para poder efetuar a marcação dos parâmetros de acordo com o seu input. Para explicar mais detalhadamente como este sistema de pistas funciona, vamos a um exemplo.

Lightfoot (1999) mostra que nas línguas V2, ou seja, nas línguas que exigem que o verbo finito esteja em segunda posição em sentenças simples, se um XP se move para Spec, $\mathrm{CP}$ e o verbo finito não sobe para $\mathrm{C}^{\circ}$, a sentença é agramatical. Sabe-se que esse XP pode ser um sujeito, um objeto direto, agente ou paciente, etc. Assim, o que se necessita é de uma condição da GU que exija que o material lexical presente no especificador de $\mathrm{CP}$ precise ser licenciado por um $\mathrm{C}$ preenchido lexicalmente. Uma alternativa, seguindo Uriagereka (1988), seria, nas palavras de Lightfoot (1999:153): "que IP é uma barreira para extração, ao menos que ela seja regida por um $\mathrm{C}$ lexical. $\mathrm{O}$ único núcleo que pode se mover para $\mathrm{C}$ sem violar as condições usuais sobre movimento de núcleos (Aoun et al. (1987); Rizzi (1990)) é um verbo, movendo através de I e, portanto, tomando traços de finitude. Isso dá origem à estrutura abaixo, e nós explicamos por que o verbo finito deve mover para $\mathrm{C}$ :

$$
\operatorname{SpecCP}[X P] C^{[}[\mathrm{Vf}]{ }_{\text {IP }}[\ldots
$$

Se esta análise estiver correta, então o que a criança que está adquirindo uma língua V2 tem de aprender é que os enunciados começam com uma categoria sintagmática arbitrária. O resto da informação vem da GU. Portanto, a pista é (6) [= (13)], uma estrutura abstrata e um elemento da língua- $I$ :

$$
\left.5_{\text {Spec CP }} X P\right] . "
$$

No entanto, se esse XP for o sujeito da oração, a criança poderá atribuir duas análises para a sentença: uma em que o XP está em spec,IP e outra em que o XP está em spec,CP. Como este tipo de construção não fornece evidências inequívocas para a criança, ela não será considerada uma pista, ou seja, as pistas serão aquelas estruturas não ambíguas, que só podem receber uma única análise. Portanto, no caso das línguas V2, a pista 
será uma estrutura como a em (13) acima, cujo XP não é sujeito da sentença. Lightfoot reporta que por volta de $30 \%$ dos enunciados em holandês, por exemplo, são constituídos por estruturas deste tipo, com um XP não sujeito em spec,CP. Portanto, o autor assume que as pistas devem ser dados robustos, salientes no input da criança. ${ }^{7}$

Assumindo tal teoria de aquisição como válida e baseando-me na generalização de Cheng apresentada acima, assumirei que esta generalização está, de alguma forma, presente na GU. Desse modo, a criança, ao iniciar o processo de aquisição de uma língua, procurará por uma pista que indique se sua língua gera perguntas QU através de movimento ou não. Como as línguas de QU in situ sempre terão uma partícula interrogativa em $\mathrm{C}^{\circ}$ nas perguntas $\operatorname{sim} /$ não, ${ }^{8}$ a criança, então, terá de procurar pela seguinte pista:

\section{$\left[C_{C^{\circ}}\right.$ partícula interrogativa $]$}

Se a criança encontrar este $C^{\circ}$ preenchido por uma partícula, ela marcará sua língua como sendo [-movimento QU]. Se, por outro lado, ela não encontrar este núcleo preenchido, ela marcará sua língua como [ + movimento QU]. Note que (14) se qualifica perfeitamente como sendo uma pista, uma vez que ela é não-ambígua e, nas línguas de QU in situ, ela é bastante saliente no input, já que todas as perguntas sim/não possuirão este elemento.

No caso do $\mathrm{PB}$, a criança, ao iniciar o processo de aquisição, não encontrará $\mathrm{C}^{\circ}$ preenchido com uma partícula e, por isso, marcará o parâmetro como [+movimento QU]. Com o parâmetro fixado, todas as suas perguntas conterão um elemento interrogativo deslocado. De fato é isto o que ocorre: até os 3;9 de idade, todas as interrogativas de N. possuem o elemento QU na periferia esquerda da sentença.

No entanto, existe um momento em que a criança começará a produzir, além de interrogativas com QU deslocado, interrogativas com QU in

\footnotetext{
7 Após analisar a porcentagem de freqüência de algumas outras pistas, Lightfoot assume que um número entre 17 a $30 \%$ de freqüência de pistas no input seria suficiente para que a criança pudesse acessá-las e, consequentemente, marcar o parâmetro correspondente.

8 Note que, nas línguas de QU in situ, as perguntas QU podem não apresentar tal partícula interrogativa em $\mathrm{C}^{\circ}$. Portanto, a criança tem de procurar por esta pista em perguntas que sempre apresentam tal partícula, ou seja, as perguntas sim/não.
} 
situ. Temos que assumir então que a marcação deste parâmetro é, de alguma forma, alterada durante a aquisição do $\mathrm{PB}$, já que a marcação $[+$ movimento QU] não permite a geração de QU in situ.

Para que a criança mude a marcação deste parâmetro, é necessário que ela perceba que, no input a sua volta, perguntas-QU sem movimento são produzidas. Ou seja, é necessário que haja uma modificação da percepção da criança sobre o seu input. Proponho aqui que é a existência de estruturas como as abaixo que desencadeiam esta mudança de percepção:

(15) a. Quem que você gosta $\left[_{\mathrm{PP}} \mathrm{cv}\right]$ ?

b. O que você vai precisar $\left[_{\mathrm{pp}} \mathrm{cv}\right]$ pra fazer o bolo?

c. Esse livro eu vou precisar $\left[_{\mathrm{PP}} \mathrm{Cv}\right]$ pra semana que vem.

d. O João eu gosto $\left[_{\mathrm{pP}} \mathrm{cv}\right]$.

As sentenças em (15) possuem uma categoria vazia 'especial', que não possui conectividade categorial com o elemento QU (ou o tópico) presente na periferia esquerda co-referente a ela. Observe que o elemento na periferia esquera é um DP ao passo que o verbo seleciona um PP como complemento. Essa ausência de conectividade categorial me leva a assumir que nestas construções não há movimento do elemento QU (ou do tópico) para a periferia esquerda da sentença, sendo que esse elemento é gerado na base na posição de spec,FocP (ou TopP). Assim sendo, a criança, ao aprender que os verbos gostar e precisar subcategorizam um $\mathrm{PP}$ e ao notar que o elemento interrogativo (ou o tópico) é um DP, também assumirá que tais elementos presentes na periferia esquerda são gerados na base e que não há movimento nesses casos. Esse tipo de análise fará com que a criança questione a marcação do parâmetro [ + movimento QU], uma vez que ela estará diante de estruturas que não foram geradas via movimento. A pista para a criança neste caso seria (16):

$$
\left.\left[\mathrm{vPP}_{\mathrm{VP}} \ldots \mathrm{E}_{\mathrm{V}} \mathrm{V}\right] \ldots \mathrm{cv}_{\text {especial }} \ldots\right]
$$

Observe que esta pista se qualifica como tal, uma vez que ela é nãoambígua ${ }^{9}$ e bastante freqüente em PB adulto. A identificação desta cate-

\footnotetext{
9 Uma análise alternativa para as sentenças em (15) seria assumir que o PP se move da posição de complemento do verbo para a periferia esquerda e posteriormente há um apagamento desta preposição. No entanto, não teríamos como explicar, primeiramente, como se dá esse apagamento e em segundo lugar, porque esse apagamento somente ocorreria nos casos de movimento.
} 
goria vazia especial será suficiente para que a criança note a presença de estruturas geradas sem movimento e fique atenta para a marcação feita inicialmente de que sua língua é [+movimento QU]. Até então a criança era, digamos, 'surda' para as construções envolvendo QU in situ em seu input, uma vez que a marcação do PB como [ + movimento QU $]$ impedia uma completa análise dessas sentenças por parte da criança. Ao notar que essa marcação pode estar equivocada, a criança começa a ser capaz de 'analisar' (ingl. 'parse') essas sentenças. Com isso, a criança se vê defronte a duas possibilidades: (a) mover um elemento QU, como ela tem feito desde as primeiras perguntas QU produzidas ou (b) deixar tal elemento QU in situ, como ela acaba de perceber no input que é possível. Nesse momento a criança muda a marcação do parâmetro de [+ movimento QU] para [ \pm movimento QU], que seria a marcação correspondente à do $\mathrm{PB}$ adulto.

Note que com esta proposta não estou assumindo que é a presença da categoria vazia especial que faz com que a criança mude automaticamente a sua marcação inicial do parâmetro. O processo é um pouco mais complexo e não automático, e o que eu estou propondo é que, com a presença dessa categoria vazia especial, a percepção da criança sobre o seu input é modificada. É a partir desta mudança que a criança notará a possibilidade de gerar estruturas em dependências-A' sem lançar mão de movimento para a periferia esquerda da sentença. Com esta análise explicamos porque a criança começa o processo de aquisição do $\mathrm{PB}$ com um parâmetro e, no meio desse processo, modifica a marcação inicial. É importante frisar que essas mudanças foram motivadas sem a necessidade de postulação de evidência negativa ou pistas pouco salientes no input, o que está de acordo com o espírito da teoria adotada.

Antes de finalizar esta seção, gostaria de comentar sobre um potencial problema para a proposta formulada aqui. Dresher (1999) afirma que a estratégia de aprendizagem a partir de pistas seria 'determinística', ou seja,

Uma outra alternativa também problemática seria considerar que a preposição ocorrendo na posição de complemento do verbo é nula. Como explicar então que ela só pode ocorrer com elementos não realizados fonologicamente? Observe as sentenças em (i):

(i) a. O João eu gosto.

b. * O João eu gosto ele.

Ou seja, teríamos que assumir uma regra ad hoc de que a preposição nula só ocorre com elementos nulos. Devido aos problemas apresentados por estas análises, considero que a melhor opção é aquela apresentada no texto e é por esta razão que assumo ser tal análise não-ambígua. 
a criança não poderia desmarcar ou desfazer a marcação de um parâmetro que já foi fixado. Isso seria necessário para que se prevenisse que o pequeno falante entrasse num 'círculo vicioso' infinito. Tal proposta é desafiada no presente estudo, uma vez que sugiro que a criança adquirindo o $\mathrm{PB}$ de fato remarca o valor do parâmetro relacionado ao movimento QU.

Para mostrar que minha proposta não fará com que a criança entre em um círculo vicioso, observo que a remarcação sugerida aqui obedece ao princípio do subconjunto, como proposto em Berwick (1985), O’Grady (1997) e Wexler e Manzini (1987):

(17) Princípio do Subconjunto (O’Grady 1997: 283):

$\mathrm{O}$ dispositivo de aquisição seleciona o valor paramétrico mais restrito consistente com a experiência.

Os valores sugeridos aqui para o parâmetro do movimento QU considerados pela criança adquirindo o PB estão numa relação de subconjunto. Isto é, o conjunto de sentenças gramaticais gerado com a marcação do valor [+ movimento QU] é um subconjunto do conjunto de sentenças gramaticais gerado com o valor [ \pm movimento QU]. Com o valor $[+$ movimento QU] apenas perguntas com QU deslocado são permitidas. Com o valor [ \pm movimento QU], tanto perguntas com o QU deslocado como perguntas com o QU in situ são gramaticais.

O princípio acima diz que o dispositivo de aquisição deve escolher o valor do parâmetro mais restrito. Isto é exatamente o que observamos na aquisição de perguntas QU em PB. No entanto, para uma criança adquirindo uma língua que tenha o valor menos restrito, é essencial que a remarcação de parâmetros seja possível. Caso contrário, a criança nunca adquiriria completamente a sua língua. Pistas presentes no input fazem com que a marcação inicial possa ser reanalizada pela criança e o novo valor do parâmetro escolhido.

Deste modo, o princípio do subconjunto exije a remarcação de parâmetros quando a língua que a criança está adquirindo corresponder a um valor menos restrito do parâmetro. As pistas aqui sugeridas não permitirão o círculo vicioso temido por Dresher, uma vez que apenas valores gerando gramáticas menos restritivas podem ser remarcados, o que não permitirá outras remarcações no caso analizado aqui. 


\section{Conclusão}

A proposta feita neste trabalho para explicar a aquisição tardia de perguntas QU in situ em $\mathrm{PB}$ salienta a importância da categoria vazia especial de que o $\mathrm{PB}$ dispõe para gerar estruturas em dependências-A' sem efetuar movimentos. Basear tal proposta na assunção de que é essa categoria vazia especial do $\mathrm{PB}$ que faz a criança mudar a sua percepção sobre o input traz a vantagem de explicar porque não haverá essa mudança paramétrica (de [+ movimento QU] para [ \pm movimento QU]) em outras línguas que não dispõem desta categoria vazia. Além disso, esta análise prevê que a aquisição de tal categoria vazia especial será anterior à aquisição de QU in situ. Esta previsão é comprovada nos dados de N., uma vez que a aparição da primeira construção similar às mencionadas em (15) (que apresentam esta categoria vazia especial) é aos 2;9 de idade, e a primeira aparição de QU in situ acontece somente aos 3;9 de idade.

Esta análise nos permite fazer ainda uma outra previsão no que concerne à aquisição de QU in situ nas línguas estudadas por Cheng que possuem QU in situ opcional: o árabe egípcio, o bahasa indonesia e o palauan. Como nestas línguas existe uma partícula interrogativa em $\mathrm{C}^{\mathrm{O}}$ nas perguntas com QU in situ, a criança deverá marcar o parâmetro como [- movimento QU] e, desde suas primeiras produções de perguntas QU, ela deverá deixar o elemento interrogativo em sua posição argumental. A aquisição de perguntas com o elemento QU na periferia esquerda da sentença deverá ocorrer somente mais tarde, pois dependerá da aquisição das sentenças clivadas de onde tais perguntas são derivadas.

Este estudo traz uma idéia geral de como os dados de aquisição podem ser úteis para comprovar ou refutar uma teoria. O comportamento lingüístico de $\mathrm{N}$. foi crucial para que pudéssemos analisar não só seus enunciados, como também oferecer uma análise alternativa para o PB adulto. Pudemos classificar o PB adulto como uma língua $[ \pm$ movimento QU] e os enunciados da criança fornecem suporte a esta análise.

Recebido em dezembro de 2003 Aprovado em julho de 2004 


\section{REFERÊNCIAS BibliográFiCAS}

Aoun, Joseph, Norbert Hornstein, David Lightfoot e Amy Weinberg. 1987. Two Types of Locality. Linguistic Inquiry 18: 537-577.

BERWICK, Robert. 1985. The Acquisition of Syntactic Knowledge. Cambridge, MA: MIT Press.

Cheng, Lisa. 1991. On the Typology of WH-Questions. Tese de Doutorado, MIT.

Chomsky, Noam. 1995. The Minimalist Program. Cambridge, MA: MIT Press.

e Howard LasNiK. 1977. Filters and Control. Linguistic Inquiry 8: 425-504.

Dresher, Elan. 1999. Charting the Learning Path: Cues to Parameter Setting. Linguistic Inquiry 30: 27-67.

e Jonathan KAYE. 1990. A Computational Learning Model for Metrical Phonology. Cognition 34: 137-195.

FoDOR, Janet. 1998. Unambiguous Triggers. Linguistic Inquiry 29: 1-36.

Grolla, Elaine. 2000. A Aquisição da Periferia Esquerda da Sentença em Português Brasileiro. Dissertação de Mestrado, IEL, Unicamp.

Kato, Mary e Eduardo Raposo. 1994. European and Brazilian Portuguese Word Order: Questions, Focus and Topic Constructions. Trabalho apresentado no Linguistic Symposium on Romance Languages XXIV, Washington, DC.

Lightfoot, David. 1999. The Development of Language: Acquisition, Change and Evolution. Massachusetts: Blackwell Publishers Inc.

Lopes-Rossi, Maria Aparecida. 1996. A Sintaxe Diacrônica das Interrogativas-Q do Português. Tese de Doutorado, IEL, Unicamp.

O'Grady, William. 1997. Syntactic Development. Chicago: The University of Chicago Press.

RızzI, Luigi. 1990. Relativized Minimality. Cambridge, MA: MIT Press.

SiKANSI, Nilmara. 1999. A Aquisição das Interrogativas-Q do Português do Brasil. Qualificação de Tese de Doutorado, IEL, Unicamp.

Uriagereka, Juan.1988. On Government. Tese de Doutorado, University of Connecticut.

Wexler, Kenneth e Maria Rita MAnzinI. 1987. Parameters and Learnability in Binding Theory. In: T. Roeper e E. Williams (eds.) Parameter Setting, 41-76. Dordrecht: Reidel. 